\title{
Interactive comment on "Deeply subducted continental fragments: I. Fracturing, dissolution-precipitation and diffusion processes recorded by garnet textures of the central Sesia Zone (Western Italian Alps)" by Francesco
}

\section{Giuntoli et al.}

\section{Anonymous Referee \#1}

Received and published: 29 September 2017

Summary statement: The manuscript submitted by Giuntoli et al. attempts to contextualize the formation of garnet microstructures and chemical zonation in relation to subduction processes. The manuscript aims to unravel the polymetamorphic and deformation history of the metasedimentary eclogites from the Western Alps. The work presents quality analytical data on garnet zonation and attempts to explain the processes that lead to their formation. Its premise is a beneficial contribution to understanding microscale chemical processes and the dataset is extremely nice. Unfortu- 
nately, the data is presented without sufficient context to allow the reader appropriate information to assess the processes proposed. This renders the main conclusions regarding multiple Alpine metamorphic events unjustified, or extremely difficult to support based on the uncertainties within the data set. The manuscript requires the presentation of the complete methods utilized, together with greater contextualization of the behaviour of other minerals within the rock to appropriately understand garnet and therefore eclogite evolution. I understand that some of this information is presented in second installment or in different papers, if the data is reutilized it is not appropriate and leaves this manuscript with no novel quantitative information. This needs to cleared up and explicated stated, as current it is confusing and ambiguous. If this the case the paper is unacceptable for publication in its current form.

Specific comments: The main conclusions ascribing two periods of garnet growth/resorption during the high-pressure Alpine metamorphism is largely unconvincing. The $P$ estimates established for rims 2 and 3 are extremely similar, and within uncertainty estimates shown in Figure 8b. It is unknown how these estimates where determined outside of using a program GRTMOD making them hard to believe. What thermobarometry did the program use? What uncertainties are there on the method? What equilibrium volumes were assessed? This information together with the presentation and discussion of the complete thermodynamic models used, and their parameters is needed to justify the estimates provided. The presentation of pseudosections would also provide greater context to the history of the rocks. In particular, the questions surrounding what reactions are controlling garnet growth/resorption? what is the shape and habit of its isopleths? what part of the rock volume was equilibrated? These become difficult to assess in its current form, particularly by the assertion of fluid ingress. The influence on fluid needs to be discussed more fully in terms of the modelling volumes. If the equilibrium volumes are dependent on the diffusion of certain elements additional modelling of chemical potential gradients would be of potential benefit. 
In terms of the textural evolution of garnet, greater context of the surrounding phases is required to completely assess the rocks evolution, from both a microstructural and chemical perspective. The sampling is from diverse compositions and protoliths, but consideration of the similarity of the processes is not adequately provided. It begs the explanation of how the period of brittle fracturing is linked to any remnant of current fabrics and their prevailing strain distribution locally and regionally? This manuscript largely states the processes of dissolution and precipitation without providing sufficient evidence to support and disprove possibilities. Presentation of quantitative microstructural information would greatly improve the submission and support some of the conclusions. Much of the focus is on garnet chemistry and not the rocks microstructure.

These issues require contextualization and transparency to support and convince the reader of the interpretations. Many sentences are unclear or have grammatical errors that make it difficult to be sure what the author are trying to convey.

Technical corrections: Page 1 Line 20: “...Sesia Zone, with a general decrease in fluid-garnet interaction observed towards the external areas"

Line $22: 100 \%$ of what?

Line 23: what deformation structures? need to look at other phase than just garnet, and even then, you have only talked about brittle fractures. What about crystal plasticity?

Page 2 Line 2: may alter the zonation recorded by garnet

Line 3: diffusion is also dependent on the medium or deformation i.e. grain boundary fluid or pipe diffusion

Paragraph 2: the introduction needs a clearer statement about what the manuscript is trying to solve

Line 18: Permian high-temperature and Alpine high-pressure events. How have these events been determined in age? 
Line 19: minerals shouldn't be pluralized

Line 20: clumsy sentenced would benefit from rewording

Line 32: this is an interpretation and needs to be stated as such

Line 33: jumps to generalities of garnet growth from specifics of Alpine rocks, needs to be clearer and explicit following one logic flow then the next

Interactive

comment

Page 3 Second paragraph: at this stage, it is not clear how you propose to evaluate these possibilities, and exclude different scenarios that lead to your preferred interpretation

Line 9 and 10: confusing sentence needs rewording. As it reads it seems you collected the samples away from the foliation?

Line 11: delete single

Line 12: is the quantitative data already published? This is a major flaw and is not appropriate.

Line 23: delete but

Page 4 Line 3: overprints Line 4: granulite facies conditions and retrograde amphibolite facies conditions

Line 13: latter? Be explicit

Line 15 replace were with involved

Line 22: present the end member formulae used throughout

Line 24: ... representative amounts of the samples

Printer-friendly version

Line 30: quantify size range

Page 5 I think the use of rim doesn't really apply, they are interpreted growth zones.

Using rim makes distinguishing the processes and stages difficult, as in most instance 
they are not physically rims. The microstructures, need more detailed descriptions and not left in the supplement. Grains sizes should be quantified. Requires photomicrographs

Line 2: are these exsolution needles? If so this could be of significance to temperature or pressure estimates

Line 6: from the internal and external areas

Interactive

comment

Line 23: sentence needs rewording

Line 30: I am not sure this is completely true, EBSD provides quantitative information about garnet microstructures, X-ray maps provide links between chemistry and textures. Deleting the word most would help this sentence.

Page 6 Line 1: change pictures to images

Line 10: How was the sealed fractures accounted for in the fractionated compositions and the modelling procedures?

Line 16: how is this accounted for? Growing in two directions

Line 19: providing some quantification of the growth scale in particular directions would strengthen conclusions, particularly across the diverse samples. However this does not explicitly consider crystallography

Line 24: Almandine and spessartine vary a lot in the cores?

Page 7 Line 2: the sealing of the fracture pattern is not obvious on the element maps?

Line 10: again, not obvious what this is talking about; the pyrope contents are unevenly distributed why is this so?

Line 17: remove interpretative statements in the results section

Paragraph 3 No results for the thermodynamic modelling are presented, this is more so thermobarometry. The models should be shown and the methods used to estimate 
P-T discussed. What parts of the garnet were fractionated (supplement figure maybe), how did this vary throughout the rest of the rock volume, garnet does not grow in isolation. No equilibration context is provided, it would be good to know the reactions controlling the growth/resorption. What are the nature of the garnet isopleths? Was fluid added and how did it change the modelling? These questions are not explicitly addressed. Discussion of the uncertainties of the P-T estimates are fundamental to resolving these discrete events, currently they are within errors presented in Figure 8 and cannot be resolved. The way this reads it seems the data has been presented elsewhere and therefore is largely re-interpreted and not appropriate as central conclusion of this manuscript, what is new here?

Page 8 Line 2: don't start a sentence with and

Line 7-14: there is an extremely large variation in pressure across all samples, encompassing the entire range of the high-pressure event, how can these be resolved as discrete metamorphic episodes?

Line 17: there is no $\mathrm{U}-\mathrm{Pb}$ ages presented?

Line 30: replace fillings with more indicative terminology

Page 9 Line 1: veins? Keep terminology consistent

Line 4: best observed, delete visible. But less so for Mn

Line 5: if concentration limits are near the lower limits of detection this should be discussed in the results

Line 6: yes, grossular is commonly pressure dependent, but this should be show specifically for these samples together with the nature of garnet modes to assess potential resorption.

Line 8: sentence needs restructure as it is confusing in its current form

Line 9: these options should be presented as possibilities unless direct evidence is 
shown to support different possibilities. Sentence also jumps from small-scale to large earthquake features

Line 10: referencing an in-preparation manuscript is bad practice and not appropriate and should be removed. Is there evidence to support difference in rheology to the matrix phases?

Line 15: how do you know that fluid was introduced? Needs some direct evidence

Lines 18-26: some of the fractures are accompanied by distinct Xalm and Xsps contents comparatively to the high-pressure rims, how is this accounted for under a highpressure and not a low-pressure event. Presentation of modelling would aid this discussion

Line 29: add the, before the word most

Page 10 Line 1: what other possibilities could there be that could be discounted?

Line 3: some of these zones are barely present

Line 8: present the evidence to support this and discount the other possibilities, otherwise it feels a bit like special pleading

Line 31: this possibility should be discussed in light of other potential ways to resorb garnet, i.e. P-T paths involving consumption. This becomes impossible to evaluate as the modelling is not shown and it is unknown if water was added or subtracted from the modelling

Page 11 Line 10: best observed

Line 14: if this is re-equilibrating, what phases is it adjusting with? i.e. what is the volume and how can the P-T be robust if the equilibrium volume is a few microns within a garnet grain with introduced fluid

Interactive

comment

Page 12 
Line 7: the mineral equilibria modelling has not been shown and the paragenesis have not been outlined, these need to be discussed instead of sorely garnet chemistry

Line 10: jumps to the gross scale. A clear picture of the relationships of the different samples and their degree of change should be provided to aid the reader

Page 13 Line 24: this study does not show or discuss zircon, so how can we know it has brittle deformation features in its cores?

Line 33: if the diffusion of the different element influenced the re-equilibration volumes, then discussion and presentation of chemical potential gradients would aid the understanding of the potential re-equilibration processes

Figures Figure 1. If all samples are from the internal complex how can it be known what the difference in ingress occurred in the external complex?

Figure 2: should be all photomicrographs to discuss the texture then presentation of chemical maps. Additional photomicrographs of the general textures observed throughout the samples would provide better context to the garnet microstructure and evolution of the samples.

Figure 3: in many of the captions, interpretations are presented as facts. Interpretations should be avoided wherever possible in figure captions

Figure 6: avoid interpretations in the caption, especially surrounding re-equilibration it is not known what proportion of the rock volume is in equilibrium

Figure 8: where is the data from? How was it collected? if the data is from a previous publication this is inappropriate. What method was used to determine the P-T? what are the uncertainties? The high-pressure event overlaps suggesting any discrete events are not resolvable

Figure 9: This figure is inconsistent with Figure 8. How is rim1 formed at low-pressure but it is present as a high-pressure stage? Rim2 and rim3 are within error and likely 
reflect one in the same process. The garnet modes have no context without the pseudosection, how is it know the water was introduced specifically at this time, was this SED incorporated into the model parameters?

I hope these comments help

Interactive

Interactive comment on Solid Earth Discuss., https://doi.org/10.5194/se-2017-87, 2017.

comment 